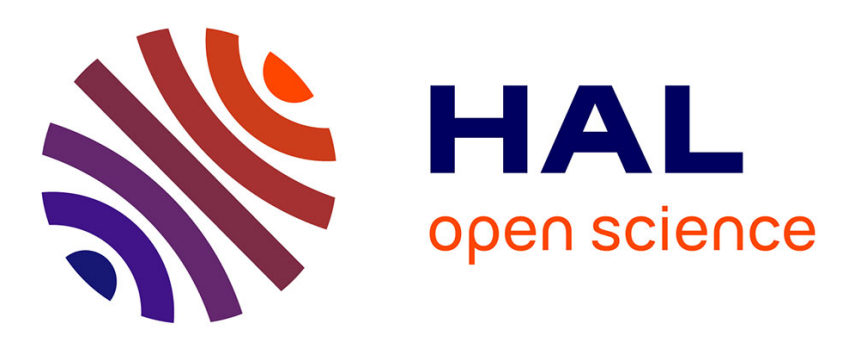

\title{
How morphology of artificial organisms influences their evolution
}

\author{
Nikolai Bessonov, Natalia Reinberg, Vitaly Volpert
}

\section{To cite this version:}

Nikolai Bessonov, Natalia Reinberg, Vitaly Volpert. How morphology of artificial organisms influences their evolution. Ecological Complexity, 2015, 10.1016/j.ecocom.2015.09.005 . hal-01237505

\section{HAL Id: hal-01237505 \\ https://hal.science/hal-01237505}

Submitted on 8 Dec 2015

HAL is a multi-disciplinary open access archive for the deposit and dissemination of scientific research documents, whether they are published or not. The documents may come from teaching and research institutions in France or abroad, or from public or private research centers.
L'archive ouverte pluridisciplinaire HAL, est destinée au dépôt et à la diffusion de documents scientifiques de niveau recherche, publiés ou non, émanant des établissements d'enseignement et de recherche français ou étrangers, des laboratoires publics ou privés. 


\title{
How morphology of artificial organisms influences their evolution
}

\author{
N. Bessonov ${ }^{1}$, N. Reinberg ${ }^{1}$, V. Volpert ${ }^{2}$ \\ ${ }^{1}$ Institute of Problems of Mechanical Engineering, Russian Academy of Sciences \\ 199178 Saint Petersburg, Russia \\ ${ }^{2}$ Institut Camille Jordan, UMR 5208 CNRS, University Lyon 1, 69622 Villeurbanne, France
}

\begin{abstract}
The purpose of this work is to study virtual populations of artificial organisms with their genotype, morphology, mechanism of motion, search and competition for food, reproduction, mutations. The genotype determines the phenotype (morphology), while morphology determines efficiency of motion and success in the search for food in the competition with other individuals; sufficient amount of food allows reproduction. Ensemble of these elements constitutes the minimal model to study natural selection of artificial organisms. Considering only some of them, as it is often the case in artificial life models, can be used for the optimization of some properties (for example, robot's gait or embryo's form) but not to study natural selection in the evolutionary context.

Artificial organisms are considered in this work in the form of polygons (triangles) on the plane. Their genotype is given by three positive numbers associated to the vertices and their morphology is determined by the lengths of the sides equal the sum of the numbers in the adjacent vertices. Behavior of the individuals and their success in the search for food depend on their morphology. More efficient individuals will reproduce more than the others and will transmit their advantageous variations to their offsprings. Hence we can observe how natural selection chooses more efficient morphology and how it evolves due to random mutations.

We develop an individual based model where the individuals recognize food and move to it with the speed determined by their morphology (and not prescribed in the algorithm). If they have enough food, they survive and reproduce. Therefore morphology and evolution are tightly interconnected and should be studied together. Dynamics of such populations appears to be different from the dynamics described by conventional models of competition and evolution of species. In particular, a new phenotype can emerge due to a different strategy of foraging (related to a different morphology) and not only due to a difference in consumed resources with the existing phenotype.
\end{abstract}

Key words: artificial organisms, morphology, competition, evolution 


\section{Introduction}

The principle of natural selection implies that variations are transmitted from parents to offsprings. The individuals with advantageous variations have better fitness. Consequently, such variations spread in the population and influence its evolution. This schematic description is conventionally accepted but it jumps over an important step: how variations are related to fitness. In the other words, how the phenotype is related to the reproduction and mortality rates. It is important to note that this relation will not be imposed by the assumptions of the model but it should follow from the morphology of the artificial organisms. In order to study this question, we will introduce in this work virtual populations of artificial organisms and will observe their behavior. The main idea of this study is that we prescribe individual characteristics of the organisms (size, form) but not their behavior in the search for resources. The model presented below will allow us to study on a simple example the interaction between morphology and natural selection, or, in a more general formulation, the evolution of the phenotype.

Artificial life models. Artificial life models are largely used to study behavior of biological organisms at the individual level, their collective behavior and evolution. We will consider a complete life cycle model which includes the genotype of the organisms in its relation to the phenotype, the mechanism of motion and food search determined by the morphology of the organisms, and reproduction (Figure 1).

Genotype and phenotype. Artificial embryogeny studies the development of the organism on the basis of some set of rules considered as genotype (see [18], [24] and the references therein). These rules determine cell division, differentiation, death and possibly some other properties (motion, production of signaling molecules, etc.) resulting in the emergence of certain morphology. As a result of this development, we obtain a cellular structure which should satisfy some criteria about its form, function or regeneration [1], [4], [12].

Development of artificial organism can be considered in the evolutionary context with selection based on fitness according to some criteria (for example, to preserve homeostasis [4] or to move on longer distance [6]). In this case, natural selection is replaced by artificial selection where the organisms are not chosen according to their capacity to survive and to reproduce but according to some specific properties.

Motion. The mechanism of motion of terrestrial animals represents consecutive displacement of their members. Some of them are fixed to the ground to provide support, while some other change their position. Gait optimization is an important problem for virtual and real robots [6]. Genetic algorithms are used for this optimization with artificial selection based on some criteria [23]. The mechanism of motion is determined by the morphology of the organism and in its turn it determines the success in the search for food in the competition with other individuals.

Food search. The process of food search has the principal importance for survival and multiplication of individuals. It contains several steps: identify food, move to it and catch 


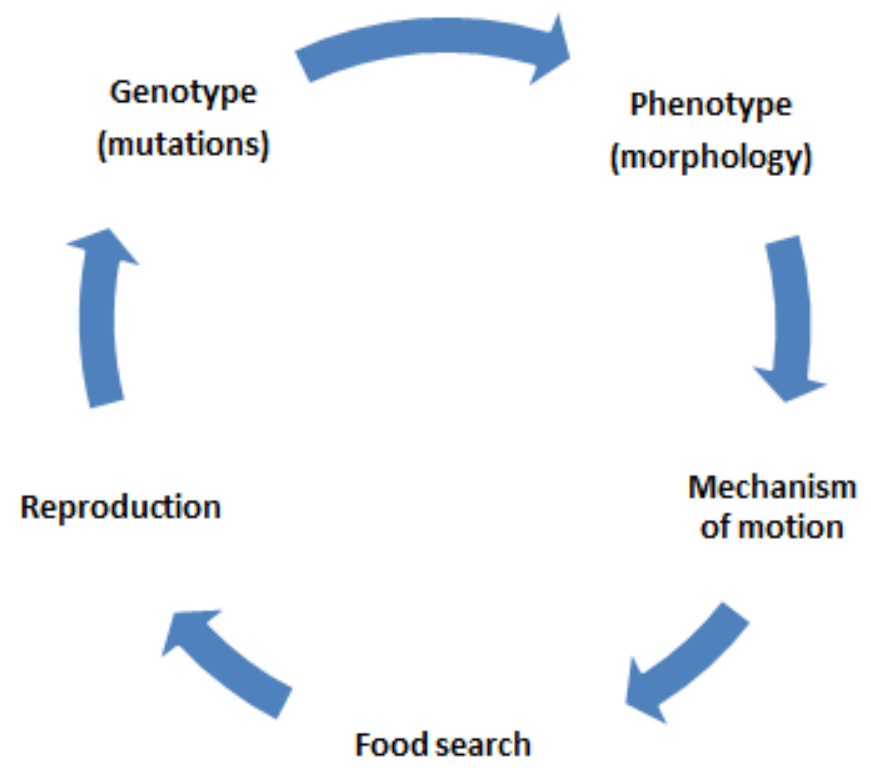

Figure 1: Schematic representation of the model. Morphology of artificial organisms determine their mechanism of motion and their efficiency in the search for food. If they have enough food, they reproduce and transmit their genotype to their offsprings with possible mutations.

it, compete with other individuals searching food. Food can be considered as a signal received by the individual through its perception system. It can be for example a visual information transmitted to the brain and identified there. Once it is recognized as food, the corresponding neurons fire and the signal is sent to the muscles. They contract and the individual starts its motion.

The signal from food and the mechanism of motion act in such a way that the individual approaches food. This assertion seems obvious but it is the most important and complex element of the process of food search. It should be stressed that there is no force here attracting the individual to the food (as in various particle dynamics models). There are neurons and muscles that react on the signal, and the mechanism of motion.

Thus we have the following chain of events: signal, neuron firing, muscle contraction, motion. Direction and speed of this motion are not prescribed. They depend on the work of neurons and muscles, and they determine how fast the individual gets to the food and whether it is more efficient than other individuals. The individuals can differ from each other by their phenotype that influences their motion.

Reproduction. More efficient individuals will get more food, will reproduce more and will transmit their phenotype (through the genotype) to their offsprings. We will consider be- 
low only asexual reproduction assuming that it is determined only by available food. The offsprings have the same genotype as parents with possible mutations. Introduction of mutations in the model will allow us to study the evolution of the phenotype.

The model developed in this work. The main goal of this work is to develop a model which includes not only some steps of the life cycle discussed above but all of them. This is important in order to describe natural selection of artificial organisms where they survive and multiply in the competition with other organisms depending on their individual properties and on the environment.

We do not characterize artificial organisms in the model by their fitness as it is conventionally done in artificial life models and in other evolutionary models. If the model imposes how fitness depends on the phenotype, then advantageous phenotypes are suggested by the model itself, and natural selection is excluded from the model.

The key property of the model developed in this work is that behavior of individuals is not prescribed. We do not impose their direction and speed of motion. They move according to their individual characteristics (phenotype), and more efficient individuals get more resources and reproduce more. In this case advantageous variations will be transmitted to offsprings and will determine the evolution of the population.

We develop a minimal model that allows us to study the phenotypic evolution due to natural selection. Each stage of the model (genotype, morphology, mechanism of motion, food search, reproduction) can be developed in a more complex and sophisticated way but this is not the goal of this work. We intend to show here the main idea of the approach to study the interaction of morphology and evolution. It opens numerous avenues for further investigation.

Artificial organisms are considered in this work in the form of polygons on the plane. We will deal here only with triangles. This is the simplest polygon that can move to the food. The interval, degenerate polygon consisting of two points, is not sufficient to insure motion to the food.

The vertices of the polygon serve as sensors that receive signals from food and act on adjacent sides changing their length. We can interpret vertices as neurons and sides as muscles, though this analogy is limited because real biological processes are much more complex.

Food is considered as points on the plane. They are inert in the sense that they do not move and do not resist. Each food point produces a signal that spreads in space uniformly in all directions and decays as a function of distance. There are three sensors (vertices) in the organism. Since the signal from food decays in space, the values of the signal received by the three sensors are in general different from each other.

The mechanism of motion consists of two half-steps. At the first half-step, the sensor (neuron), which receives the strongest signal, fires. As as result, two adjacent sides increase their length (elongation). The other two sensors do not react. The first vertex can move, the other two are attached to the ground. Hence at the first half-step, the first vertex changes its position, two other vertices are fixed. At the second half-step, the action potential of the 
first sensor (neuron) returns to its basic level, the lengths of the adjacent sides also return to their initial values (contraction). The first vertex is fixed, the two other vertices move due to the change of the side length. This mechanism of motion allows the organisms to approach the food though their motion is not directed along the shortest distance.

If the artificial organisms get enough food, then they survive and multiply. Their offsprings get the same genotype with possible mutations. The genotype of the organisms is given by three real positive numbers associated with the vertices of the polygon. The phenotype of the organism, that is the lengths of its sides, is determined by the genotype: its sides have the length equal the sum of the numbers in the adjacent vertices. Hence we determine the relation between the genotype and the phenotype. The genotype is transmitted from parents to offsprings with possible modifications of the corresponding numbers (mutations). As above, the notions of the genotype and of the phenotype are very simplified here but they retain some essential features. The genotype determines the phenotype and it is transmitted to offsprings with possible mutations.

Let us note that vertices have several functions. They contain genetic information, they also act as sensors (neurons) that recognize food and regulate the action of sides (muscles), and they participate in the mechanism of motion being either fixed or changing their position.

Phenotypic evolution. There are many open questions about phenotypic evolution (see [13], [14], [21], [22] and the references therein). Among them relation between the genotype and the phenotype, morphological constraints, emergence of new phenotypes. Some modelling approaches to the first two questions can be found in [12], [19]. In this work we will be more concerned by the third one, emergence of a new phenotype.

Among different mechanisms of speciation, the most intriguing one is sympatric speciation where the populations are not separated by genetic or geographic barriers [7], [10]. It can be described by various approaches including nonlocal reaction-diffusion equations [5], [11], [26], by individual based models and some other models [2], [3], [8], [9]. They take into account intraspecific competition that can lead to a nonuniform distribution of the population density and to the emergence of new phenotypes. One of the essential assumptions of these models is that different resources correspond to different phenotypes. Though different species (or phenotypes) can also consume the same resources and compete for them, the main parts of their resources are different. This is an important condition of their coexistence. If all individuals consume the same resources independently of phenotype, then only one phenotype persists [5], [25].

We will show in this work that different phenotypes can coexist and new phenotypes can appear even in the case if they consume the same resources. The difference in the phenotypes, which will allow them to coexist, will be related to their foraging behavior.

Thus, we suggest a model of artificial organisms with their genotype, phenotype, food recognition, side elongation-contraction, mechanism of motion, food consumption, reproduction (Figure 1). This is the minimal configuration of a biological population considered as a self-sustained system undergoing Darwinian evolution. The competition of these organisms for food will determine which of them are more efficient. We will study competition of 
different organisms and their evolution.

\section{Life and death of artificial organisms}

We consider the artificial organisms in the form of triangles on the plane. We will describe how they move, find and consume food and divide. The motion of triangles depends on their shape. So the most efficient triangles will have more food and will divide more often. New triangles appearing as a result of division can be different from their parents due to mutations. Hence we will observe natural selection and evolution of artificial organisms.

Triangle shape. We define each triangle as three points $A, B$ and $C$ on the plane. We associate with them three real positive numbers, respectively, $a_{0}, b_{0}$ and $c_{0}$. We will denote by $A B$ the side connecting the vertices $A$ and $B$, and by $L_{A B}^{0}$ its length. Similar notation is used for the other sides. We set $L_{A B}^{0}=a_{0}+b_{0}, L_{B C}^{0}=b_{0}+c_{0}, L_{A C}^{0}=a_{0}+c_{0}$. Therefore the shape of the triangles is determined by the numbers prescribed to their vertices.

External signals (food). Food is considered as a point in the computational domain. One such point is added to the computational domain each time interval $\theta$ with a random position in space. Consider a food point $D$ located outside the triangle $A B C$. We suppose that this point produces a signal that spreads in space. The value of the signal at each space point depends on the distance from this point to the point $D$. We will consider it as a given function $f(d)$ of the distance $d$. The function $f(d)$ is positive and decreasing. Everywhere in this work we set $f(d)=1 / d$.

Motion to the food. We will now describe how triangles recognize food and move to it. Points $A, B$ and $C$ receive the signal from the point $D$. The values of this signal are $f\left(d_{A D}\right)$, $f\left(d_{B D}\right)$ and $f\left(d_{C D}\right)$, where $d_{A D}, d_{B D}, d_{C D}$ are the corresponding distances. We choose the vertex, which receives the maximal signal. Suppose that it is $A$. We assume that the signal changes the lengths of the sides adjacent to the point $A: L_{A B}=L_{A B}^{0}(1+\delta), L_{A C}=L_{A C}^{0}(1+\delta)$.

Let us first describe the mechanism of motion schematically. Each motion step consists of two substeps. At the first half-step, the vertex, which receives the largest signal moves ( $A$ in our example), while two other vertices are fixed $(B$ and $C$ ). The lengths of the sides $A B$ and $A C$ increase. At the second half-step, the point $A$ is fixed, the points $B$ and $C$ can move. The lengths of the sides $A B$ and $A C$ return to their initial values. Hence the triangle returns to its initial shape but it is relocated in the direction of the point $D$ (Figure 2).

This schematic description of the mechanism of motion is pure geometrical. For its realization in the model we introduce forces exerted by the sides (muscles) on the vertices (feet) and the equations of motion. The sides of the triangle can be considered as elastic springs. In the conventional formulation, elastic spring elongates or contracts from its equilibrium length under the action of the external force. In our case, the equilibrium spring length is 


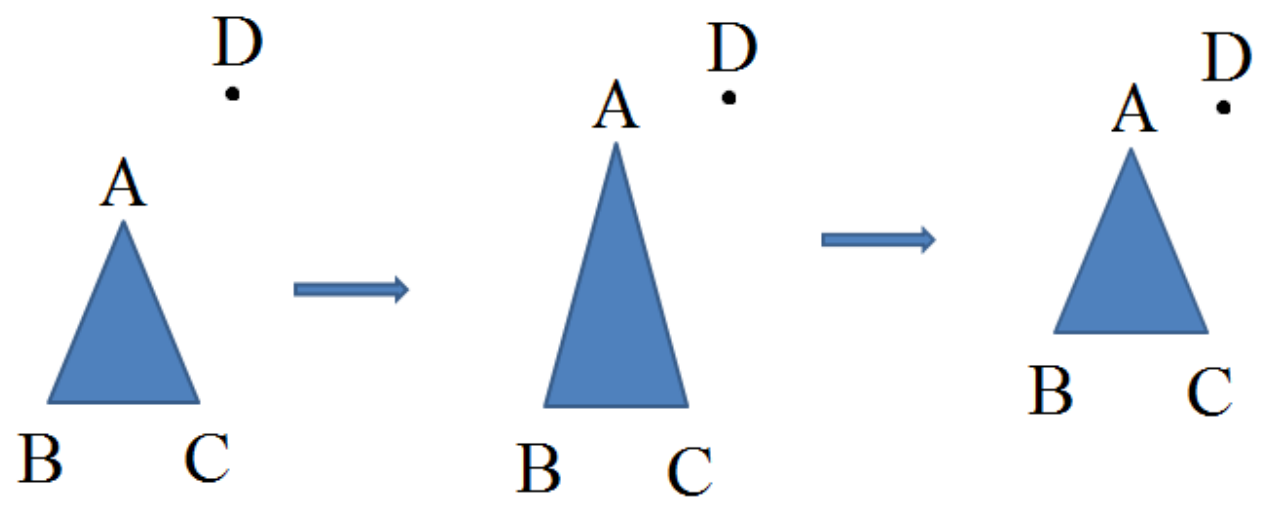

Figure 2: Schematic representation of one step in the motion of triangle. At the first step, the point $A$ moves in the direction of the source of signal, point $D$. Points $B$ and $C$ are fixed, triangle elongates (left and middle images). At the second half-step, the point $A$ is fixed, points $B$ and $C$ move, triangle returns to its original shape but closer to the point $D$ (middle and right images). The direction of motion is determined by the direction of the sides of the triangle. It approaches the source of signal but not in the shortest way.

modified as a result of the action of neuron. The force appears due to the difference between the equilibrium length and the current length of the spring.

Denote by $\vec{x}_{i}$ the coordinate vector of the point $i$ and set

$$
\vec{x}_{i j}=\vec{x}_{i}-\vec{x}_{j}, \quad \vec{x}_{i j}^{1}=\vec{x}_{i j} /\left|\vec{x}_{i j}\right|, \quad L_{i j}=\left\{\begin{array}{cc}
L_{i j}^{0}(1+\delta) & \text { first half }- \text { step } \\
L_{i j}^{0}, & \text { second half }- \text { step }
\end{array} .\right.
$$

The force acting between the vertices $i$ and $j$ is given by the formula:

$$
\vec{F}_{i j}=k\left(\left|\vec{x}_{i j}\right|-L_{i j}\right) \vec{x}_{i j}^{1} .
$$

At the first half-step, $\vec{x}_{B}$ and $\vec{x}_{C}$ are fixed and the equation of motion of the vertex $A$ writes:

$$
m \frac{d^{2} \vec{x}_{A}}{d t^{2}}=m \mu \frac{d \vec{x}_{A}}{d t}+\vec{F}_{A B}+\vec{F}_{A C}
$$

where $m$ is the mass associated to the vertex, the first term in the right-hand side describes friction by the medium. At the second half-step, the vertex $A$ is fixed and the equations of motion of the points $B$ and $C$ are as follows:

$$
m \frac{d^{2} \vec{x}_{B}}{d t^{2}}=m \mu \frac{d \vec{x}_{B}}{d t}+\vec{F}_{B A}+\vec{F}_{B C}, \quad m \frac{d^{2} \vec{x}_{C}}{d t^{2}}=m \mu \frac{d \vec{x}_{C}}{d t}+\vec{F}_{C A}+\vec{F}_{C B},
$$


Let us note that the triangle does not move in general towards the point $D$ along the shortest way, that is along the straight line connecting them. It moves along the straight line determined by the direction of the sum of the vectors $B A$ and $C A$. At some moment during this motion, the point $A$ will not be the nearest point to the point $D$ any more. Therefore another point $B$ or $C$ will receive the strongest signal and will determine the direction of motion. Thus, the triangle approaches the source of signal along some piece-wise linear trajectory (Figure 3, left).

Biological interpretation of the mechanism of motion. The vertices of the triangle have multiple functions. They keep the information about the morphology of the organism, they serve as sensors receiving signals from food, and they are connected to the mechanism of motion.

As we discussed above, each vertex contains a real positive number. These numbers determine the shape of the triangle. We will discuss below division of the triangles. The numbers in the vertices will play a role of genetic information transmitted from parents to offsprings with possible mutations.

Next, the vertices can be interpreted as neurons and the sides of the triangle as muscles. Neurons receive external signals. The neuron, which receives the strongest signal, fires and transmits the signal to muscles stimulating their action (length change). After firing, the neuron returns to its unexcited state, and the muscles return to their initial length. This analogy of vertices and sides with neurons and muscles is limited since real biological processes are much more complex. However it captures some essential features: receiving signals are transmitting action potential to target organs initiating the motion of the organism.

At the same time, the vertices of the triangle are associate with its "feet". In a more complete representation we can introduce a special organ connected to the muscles. However we avoid to complicate the model with unnecessary details. Similar to the biological organisms, the artificial organisms in the model move the feet in a consecutive manner. First, one of them is displaced with two other being fixed. After that the first foot is fixed, and two others move. Hence the artificial organism uses the friction of its feet with the "earth". Otherwise, if all vertices move at the same time when the sides elongate, the center of mass of the triangle would not move. The realization of the mechanism of motion (the choice of moving and fixed feet) can be different. However, the crucial property of the mechanism of motion, that it allows the artificial organisms to approach food (the source of signal), should be preserved.

Motion in the case of many food points. If there are many food points, then each of them produces a signal that spreads in space. Therefore each vertex of the triangle receives more than one signal. These signals are summed and they form a total signal received by the vertex. The vertex with the maximal total signal determines triangle motion similar to the case of a single signal. Since signals produced by food decay with distance, the nearest signal contributes stronger. When there are many foods, triangle moves not the nearest food but in the direction of the strongest total signal. 

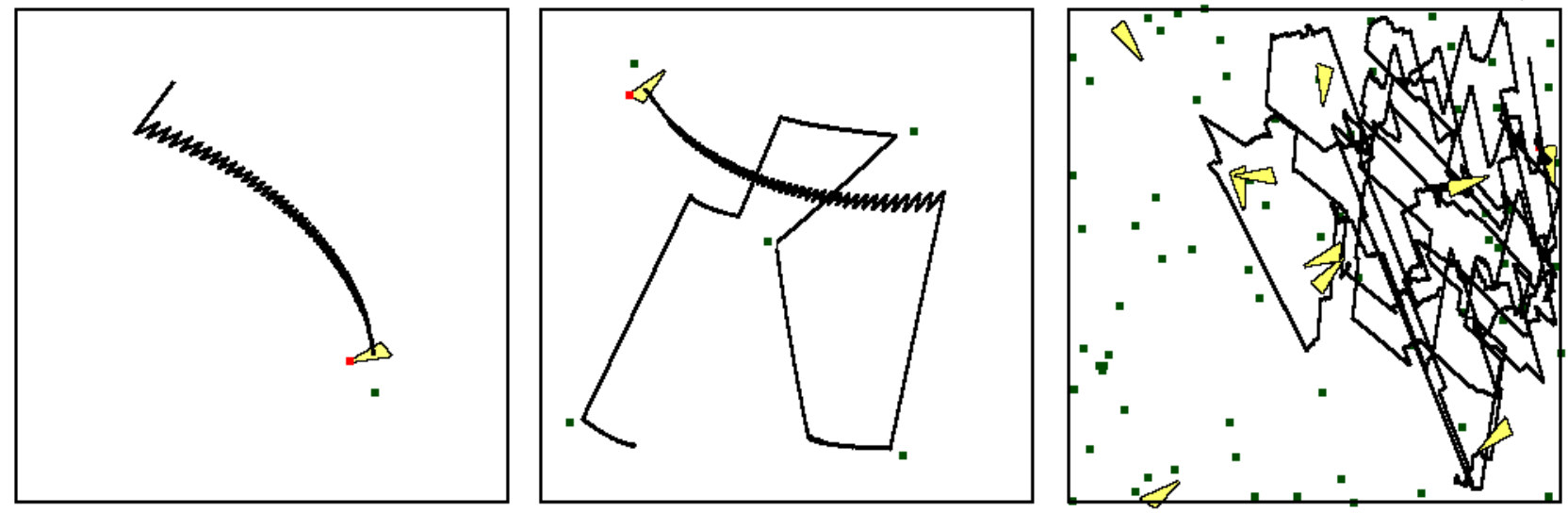

Figure 3: Triangle recognizes food and moves to it (left). When there are several food points, it first moves to the nearest one, then to the nearest among the remaining, and so on (middle). The food points are removed when they are consumed. They are kept in the figure in order to show their location. The right image shows the trajectory of one triangle in the case with many triangles and a constant food supply.

When the triangle covers the food, we suppose it is consumed. Consumed food disappears and does not produce signal any more. When all food is consumed and there is no external signal, triangles do not move. Figure 3 shows the trajectory of a triangle in the case of one and several fixed food points and in the case of a constant food supply (in the presence of other triangles).

Internal energy. Each organism possesses some characteristics which we call the internal energy and denote by $E$. If it consumes food, its internal energy grows. On the other hand, the internal energy decreases with time since the organism spends it for motion and other life processes. If the internal energy reaches some critical level $E^{*}$, then the triangle divides. If it becomes zero, it dies. Let us describe these processes in more detail.

1. Food consumption. Each consumed food increases the internal energy $E$ by 1 .

2. Energy decay. Each time interval $T=\tau / S$, the internal energy is decreased by 1 . Here $\tau$ is a given value and $S$ is the area of the triangle. Thus energy consumption depends on the area of the triangle. Larger triangles need more food to survive. Triangle area can be replaced here (and in the next property) by its perimeter (cf. Section 5.3).

3. If the internal energy reaches the level $E^{*}=\kappa S$, then the orhanism divides. Here $\kappa$ is some positive parameter. Each of the two organisms after division possess the energy $E^{*} / 2$. Other properties of newly born organisms are described below.

4. If the internal energy becomes 0 , then the organism dies. It is removed from the computational domain. 
Repulsion of organisms. There is a repulsion force $F$ between each two triangles. It is applied to the centers of mass of the triangles, and it is given by the formula

$$
F=\left\{\begin{array}{cc}
k\left(h / h_{0}-1\right) & , \quad h<h_{0} \\
0 & , \quad h>h_{0}
\end{array}\right.
$$

where $h$ is the distance between the centers of mass of two triangles, $h_{0}=\sigma\left(S_{1}+S_{2}\right) / 2$. Here $S_{1}$ and $S_{2}$ are the areas of the triangles. Thus, repulsion acts in the circle determined by the areas of triangles. Large triangle has a larger area of repulsion.

Division of organisms. Let $T_{1}$ be a triangle with the numbers $\left(a_{1}, b_{1}, c_{1}\right)$ at its vertices. When it divides, a new triangle $T_{2}$ is added. The corresponding numbers $\left(a_{2}, b_{2}, c_{2}\right)$ are given as perturbations of the numbers $\left(a_{1}, b_{1}, c_{1}\right)$ :

$$
a_{2}=a_{1}(1+\rho), \quad b_{2}=b_{1}(1+\rho), \quad c_{2}=c_{1}(1+\rho),
$$

where $\rho$ is a random variable with a uniform distribution in the interval $[-r, r]$. Hence the shape of offspring differs from the shape of parents by some random perturbations (mutations).

We need to specify the position of the new triangle with respect to the old one. We suppose that it is shifted in space and rotated on a random angle between 0 and $2 \pi$. Due to repulsion, the two triangles will go away from each other.

\section{Competition of artificial organisms}

Unstable coexistence. The speed of motion, lifespan, frequency of division of triangles depend on their morphology (shape, size). Indeed, when a triangle receives a signal from food, it elongates its two sides adjacent to the vertex, which gets the strongest signal. This elongation is proportional to the lengths of the sides: the initial length is multiplied by $(1+\delta)$. Therefore large triangles move faster than small triangles.

Moreover, the same triangle moves with different speeds in different directions because the lengths of its side are in general different from each other. If we consider its two sides as vectors and take a sum of these vectors, then this new vector determines the direction of motion. Its length is proportional to the speed of motion. Hence each triangle has three directions of motion and three speeds that correspond to these directions. Thus, the time to approach food depends on the size and on the form of the triangle.

On the other hand, triangles consume internal energy with the rate proportional to their area. Therefore large triangles need more food to survive and also more food to divide. Thus, triangles of different forms and sizes can have advantages and disadvantages. We can observe their competition and reveal the most efficient triangles.

Let us illustrate this competition with two types of triangles, equilateral and elongated (Figure 4, left). For the triangles of the first type we set $a=b=c=0.5$, of the second type $a=1.2, b=c=0.3$. We will use for them the notation $T(0.5 ; 0.5 ; 0.5)$ and $T(1.2 ; 0.3 ; 0.3)$. 

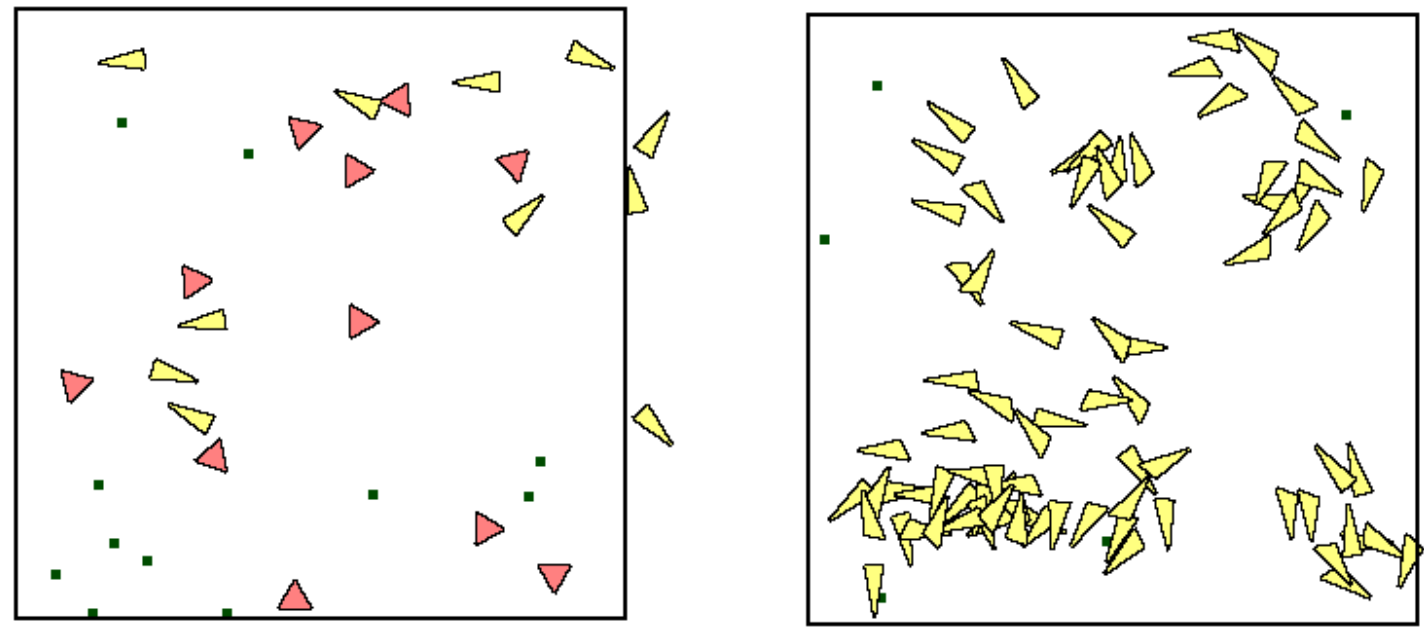

Figure 4: Competition of two triangles with the same area. Elongated triangles rapidly eliminate equilateral triangles. Initial configuration is shown in the left image, after extinction of equilateral triangles in the right image.

Their areas are approximately equal to each other. The values of other parameters are as follows: $\theta=4$ (food adding), $\tau=100$ (energy removing), $\kappa=4$ (triangle division), $\mu=4$ (friction coefficient), $\delta=0.2$ (triangle side extension). When triangles divide, their offsprings are exactly the same as their parents. We do not introduce mutations in this competition.

The initial configuration is shown in Figure 4 (left). The square surrounded by bold lines shows the area where food is added. Triangles can cross its border but they will return back in order to reach food. In the beginning there are 11 triangles of each type. After some time, equilateral triangles disappear and only elongated triangles remain. Since triangles have approximately equal areas, their energy consumption is the same and they need to reach the same level of energy to divide. Therefore the difference between them is related to their form since it determines their speed. Equilateral triangles move in all three direction with the same speed. Elongated triangles move faster in the direction of acute angle and slower in the two other directions.

It appears that triangles $T(1.2 ; 0.3 ; 0.3)$ are more efficient than triangles $T(0.5 ; 0.5 ; 0.5)$. After some time the latter disappear and only the former remain. It seems that triangles $T(1.2 ; 0.3 ; 0.3)$ are close to the optimal size and shape. They win competition with any other triangles tested in numerical simulations even if their counterparts are much more numerous in the beginning. These conclusion is confirmed for different values of other parameters.

Figure 5 (left) shows an example of competition of triangles $T(1.2 ; 0.3 ; 0.3)$ and $T(0.6 ; 0.15 ; 0.15)$. Initially, the number of triangles of the second type is 4 times greater than of the first type. However smaller triangles are rapidly eliminated. We measure proportion of triangles of these two type by the average value of the parameter $a$ associated to one of the vertices of triangles. Since $a=1.2$ for the triangles of the first type and $a=0.6$ for the 

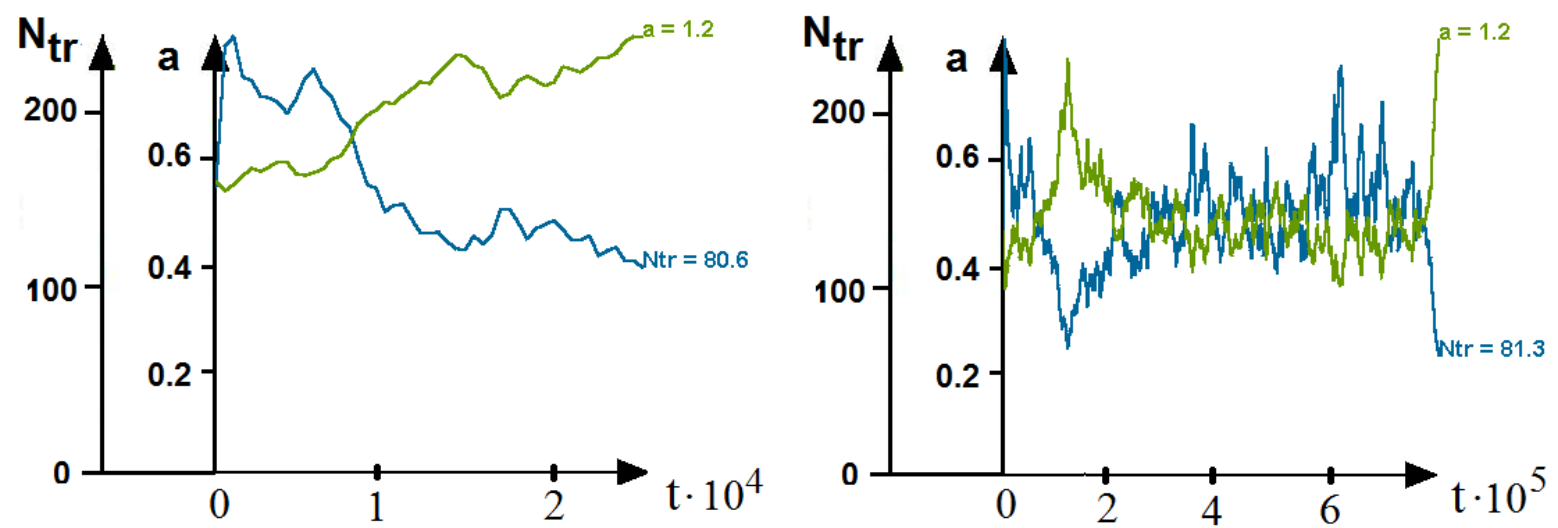

Figure 5: Competition of triangles $T(1.2 ; 0.3 ; 0.3)$ with $T(0.6 ; 0.15 ; 0.15)$ (left) and $T(1.2 ; 0.3 ; 0.3)$ with $T(0.3 ; 0.075 ; 0.075)$ (right). The curves show the number of triangles (blue) and the average value of $a$ (green) in time. The value $a=1.2$ means that only triangles $T(1.2 ; 0.3 ; 0.3)$ remain.

triangles of the second type, its average values shows their ratio. If the average value equal 1.2 , then there are only triangles $T(1.2 ; 0.3 ; 0.3)$ and the others are eliminated.

Another example of competition is shown in Figure 5 (right). We consider again triangles $T(1.2 ; 0.3 ; 0.3)$ and triangles $T(0.3 ; 0.075 ; 0.075)$ whose sides are four times smaller. Initially we put 10 large triangles and 80 small ones. They coexist during a long time but suddenly small triangles rapidly disappear.

The results of competition of triangles $T(1.2 ; 0.3 ; 0.3)$ with other triangles are summarized in Figure 6. The survival time is shown on the horizontal axis. This is the moment of time when one of triangle types disappears. The curves show the density distribution of simulations with respect to survival time. For every survival time interval $\left[\tau_{1}, \tau_{2}\right]$ we determine the proportion of simulations for which the survival time is inside this interval. This proportion represents one point of the curve. The total number of simulations for each triangle type varies from 200 to 500. All curves are normalized according to the proportion of winning triangles. If the maximum of the curve equals 1 , then triangles $T(1.2 ; 0.3 ; 0.3)$ win in all simulations. If it is less than 1 , then these triangles can also lose.

Consider the curve for triangles $T(1.4 ; 0.33 ; 0.33)$. It shows the results of the competition of these triangles with triangles $T(1.2 ; 0.3 ; 0.3)$. Since its maximum equals 1 , then triangles $T(1.2 ; 0.3 ; 0.3)$ win in all simulations. It is similar for triangles $T(0.6 ; 0.15 ; 0.15)$. If triangles $T(1.2 ; 0.3 ; 0.3)$ compete with $T(1 ; 0.25 ; 0.25)$, then they win in approximately $80 \%$ of simulations. In their competition with triangles $T(0.8 ; 0.2 ; 0.2)$ they win in approximately $95 \%$ of simulations. Therefore triangles $T(1.2 ; 0.3 ; 0.3)$ are the most efficient. Triangles with a close size can sometimes defeat them.

The dashed curve in Figure 6 shows the competition of two groups of the same triangles $T(1.2 ; 0.3 ; 0.3)$. In a big number of trials, each group wins in approximately $50 \%$ of simulations. 


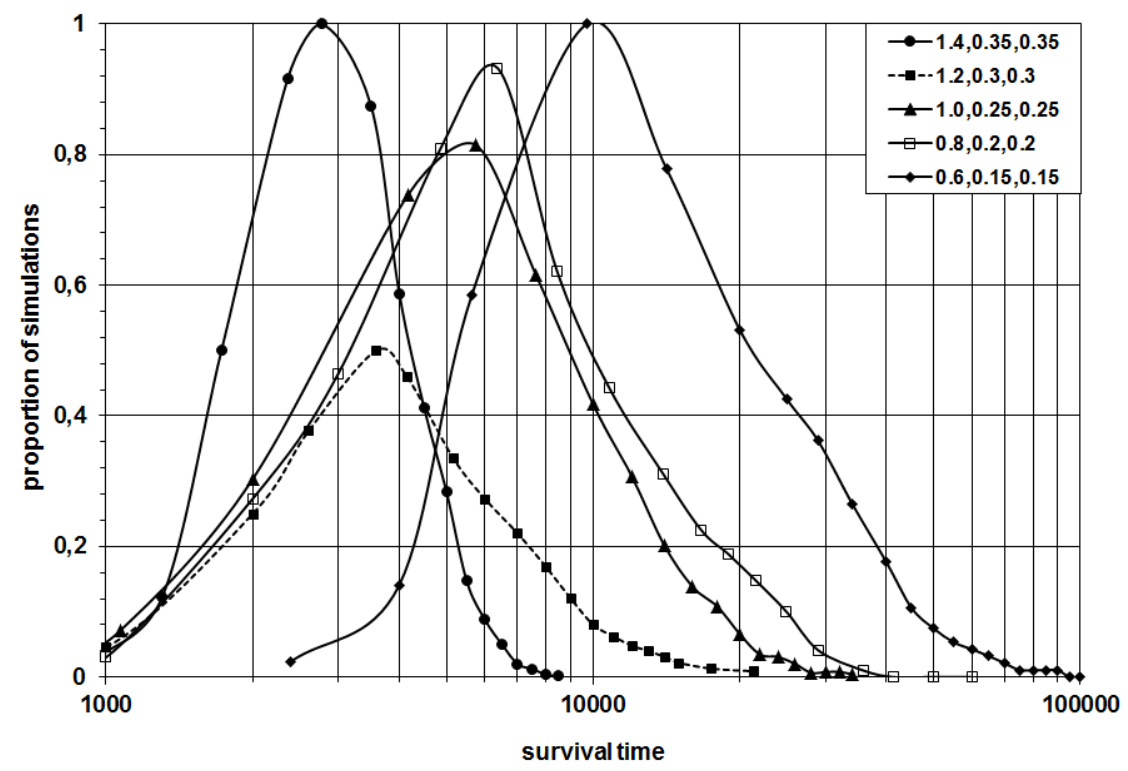

Figure 6: Competition of triangles $T(1.2 ; 0.3 ; 0.3)$ with other triangles (solid curves) and of two groups of triangles $T(1.2 ; 0.3 ; 0.3)$ (dashed curve). The curves show the distribution of simulations with respect to survival time (see the explanation in the text).

Stable coexistence. Different triangles can coexist if we increase food supply. Instead of $\theta=4$ considered above we now set $\theta=2$ : one food point is added every 2 time units.
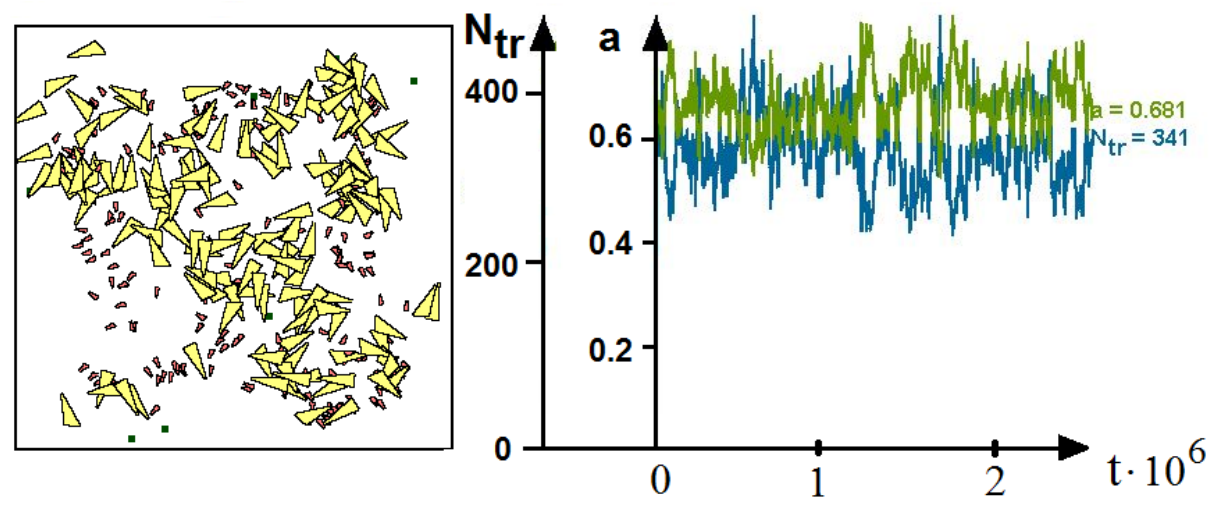

Figure 7: Competition of triangles $T(1.2 ; 0.3 ; 0.3)$ with $T(0.3 ; 0.075 ; 0.075)$ for $\theta=2$. The two types of triangles coexist. A snapshot of triangle distribution in the computational domain (left); an average value of $a$ (green curve) and the number of triangles in time (blue curve).

Figure 7 shows the results of numerical simulations of triangles $T(1.2 ; 0.3 ; 0.3)$ with $T(0.3 ; 0.075 ; 0.075)$. In the case of $\theta=4$ the coexistence is unstable and triangles $T(1.2 ; 0.3$; $0.3)$ always win the competition. In the case $\theta=2$, triangles $T(0.6 ; 0.15 ; 0.15)$ disappear 
(not shown) but triangles $T(0.4 ; 0.1 ; 0.1)$ and $T(0.3 ; 0.075 ; 0.075)$ survive and coexist with triangles $T(1.2 ; 0.3 ; 0.3)$.

Let us give a possible explanation of this result. Triangles $T_{1}=T(1.2 ; 0.3 ; 0.3)$ move much faster than triangles $T_{2}=T(0.3 ; 0.075 ; 0.075)$. When a new food point appears, the former precipitate to it and arrive there in general before the latter. However the speed of triangles $T_{1}$ is so high, that they come to the food point from a large area and their distribution in space is highly nonuniform. There are many of them around the food point and few of them farther from it. At the same time, triangles $T_{2}$ also move to the food point. Since their speed is much less, they remain far from the food. Figure 7 (left) shows the distribution of triangles $T_{1}$ and $T_{2}$ at some moment of time. This distribution is inhomogeneous in space with some areas of strong concentration of $T_{1}$ and some other areas where there are only $T_{2}$. If another food point appears now in this area, where there are few triangles $T_{1}$ and more triangles $T_{2}$, then the latter have good chances to get this food point.

This mechanism depends on the relations between the speed of motion of different triangles and the rate of food supply. This is why it works for $\theta=2$ and not for $\theta=4$. Thus, different triangles can have different strategies of getting food allowing there coexistence. It is important to stress here that they compete for the same resources and not for different resources as it is the case in conventional models of coexistence of species.

\section{Evolution of artificial organisms}

In the previous section we studied competition of different triangles in the case where offsprings have exactly the same form as their parents. We will now consider the case where offsprings can be different from parents due to small mutations. We will analyze how the phenotype changes in the process of evolution.

Let us recall that each triangle is characterized by three numbers $a, b$ and $c$ prescribed to its vertices. As we discussed in Section 2, when this triangle divides the corresponding numbers for the new triangle can differ from the previous ones due to random perturbations in a given range. Everywhere in this section we set $r=0.03$ (see Section 2 for the notation).

We begin to study evolution of artificial organisms with the initial triangles $T(1.2,0.3,0.3)$. They are the most efficient in competition with other triangles (Section 3). First of all, let us estimate the number of triangles. With their area about $S=0.4$, the time interval when the internal energy of the triangle is decreased by 1 equals $T=100 / 0.4=250$. During this time interval the number of food units supplied to the computational domain equals $250 / 4 \approx 62$. This gives us an estimate from below for the number of triangles. Since some of them will divide when they get a food unit, then their real number will be greater than this estimate. In the case without mutations their number oscillates from 80 to 83 . In the case with mutations their number oscillates between 80 and 150 (Figure 9).

Thus, oscillations of the number of triangles become much stronger because of mutations. The reason for this effect is that different types of triangles compete with each other resulting in a strong variation of their number. As we saw in the previous section, triangles $T(1.2,0.3,0.3)$ are the most efficient. So they win the competition with other triangles. 


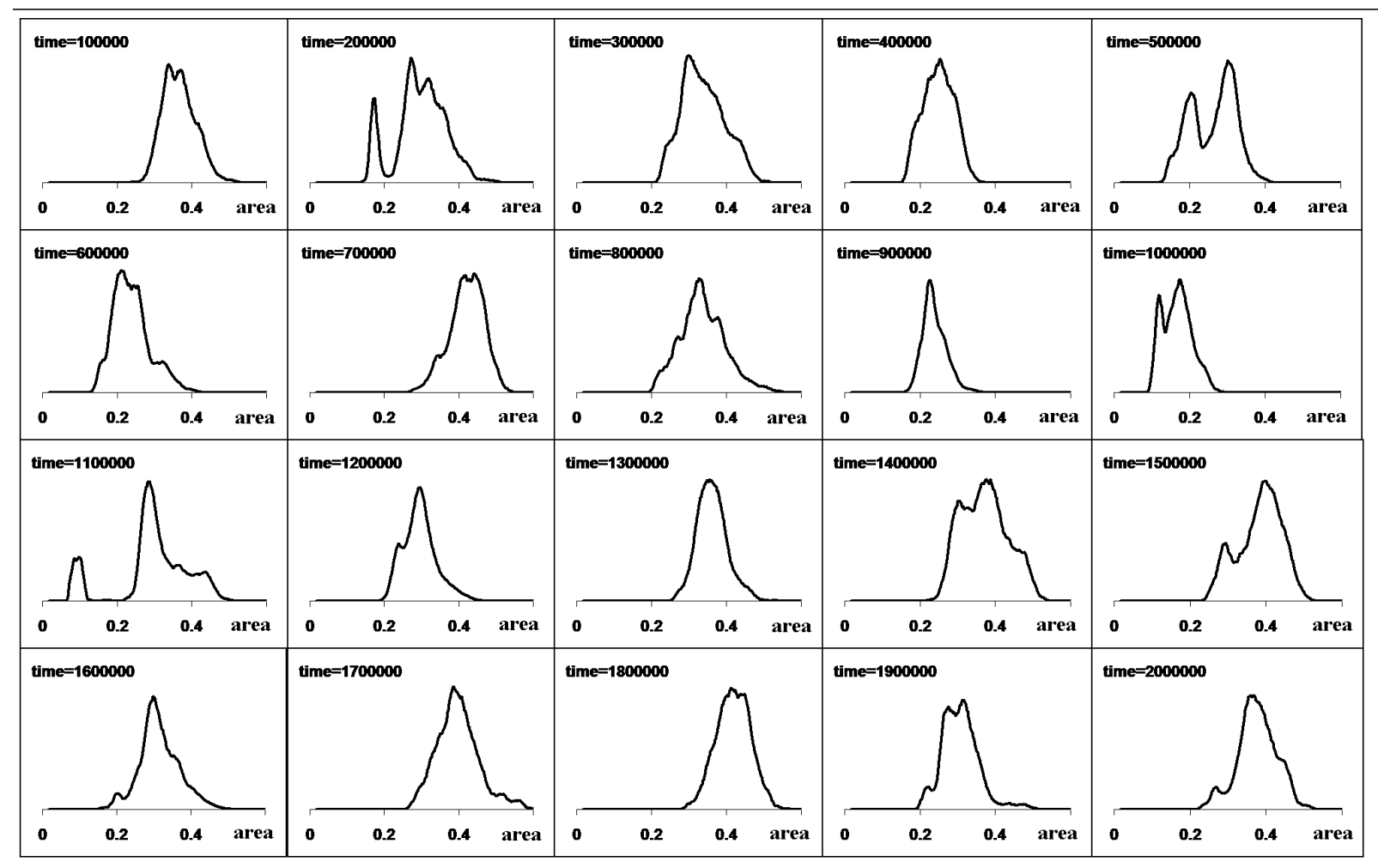

Figure 8: Evolution of triangles $T(1.2,0.3,0.3)$. The graphs show averaged distributions of triangles with respect to their area every $10^{5}$ time units.

However triangles with other forms and sizes will appear again due to mutations and this competition will start again.

The distributions of triangles with respect to their area are shown in Figure 8. The maximum of the distributions correspond to triangles $T(1.2,0.3,0.3)$. Sometimes the distributions become bimodal showing the presence of two types of triangles. Thus, if the initial triangles are $T(1.2,0.3,0.3)$, then their shape and size remain approximately constant in the process of evolution. When other triangles appear due to mutations, there is a competition between them, and triangles $T(1.2,0.3,0.3)$ win after some time.

If the initial triangles are different, then they will converge to the optimal triangles in the process of evolution. Figure 10 shows the evolution of triangles $T(1,1,1)$. The numbers prescribed at the vertices of the triangles converge to $1.2,0.3,0.3$ with some oscillations around these values. Similar behavior is observed for other initial triangles.

These results are obtained for the interval of food adding equal 4, where the coexistence of different triangles was not observed. Let us now consider this parameter equal 2. Then in the case without mutations triangles $T(1.2,0.3,0.3)$ can coexist with some other triangles $T(a, b, c)$ where $a, b$ and $c$ are sufficiently small. In particular with observed coexistence with triangles $T(0.4,0.1,0.1)$ and $T(0.3,0.075,0.075)$. 


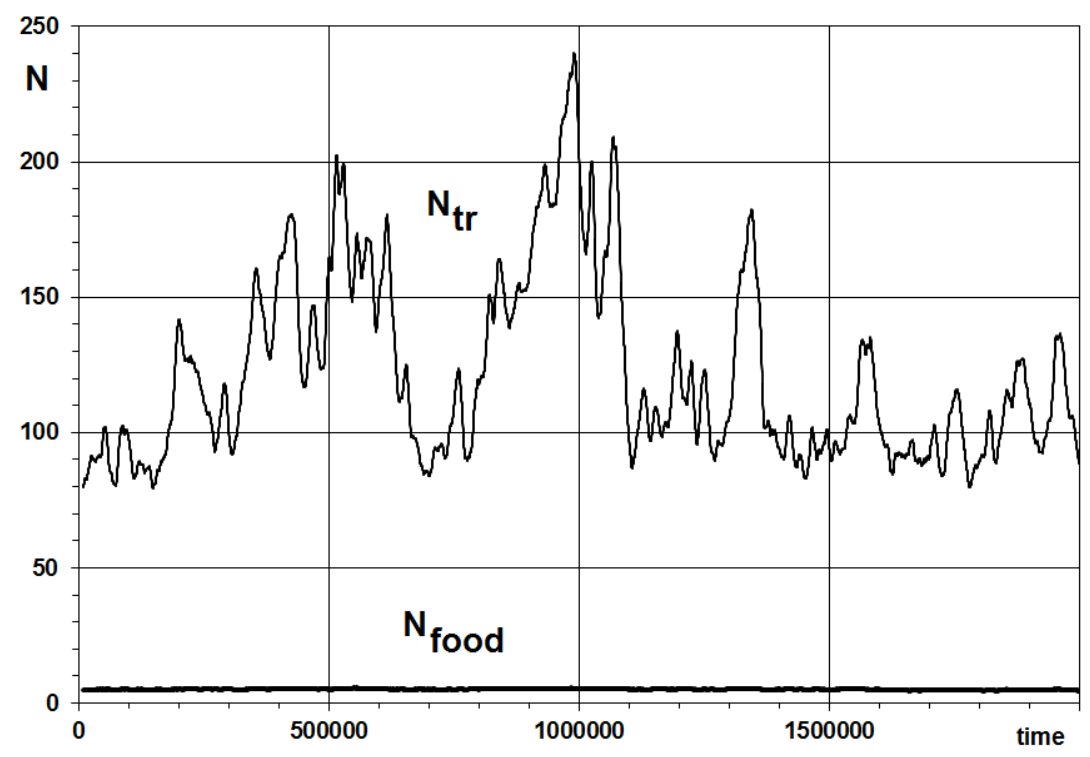

Figure 9: Evolution of triangles $T(1.2,0.3,0.3)$. The upper curve shows the number of triangles in time and the lower curve the number of food points.
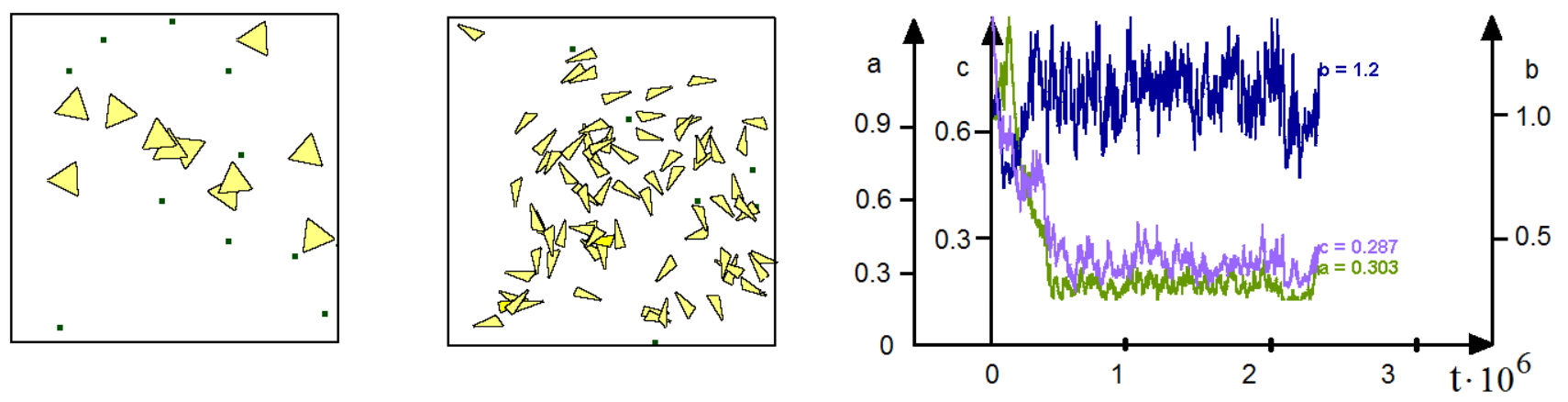

Figure 10: Evolution of triangles $T(1,1,1)$. Their initial distribution is shown in the left figure, and their distribution after some time in the middle figure. The right figure shows the evolution of the values $a, b$ and $c$ in time.

If we start the simulation with triangles $T(1.2,0.3,0.3)$, then after some time smaller triangles appear due to mutations. The distributions of triangles with respect to their area and perimeter become bimodal (Figure 11). However these distributions are not steady. Small triangles continue to decrease and their number increases. This result cannot be explained by analogy with the competition of triangles without mutations. If we consider competition between triangles $T(0.4,0.1,0.1)$ and $T(0.3,0.075,0.075)$, then the former will survive while the latter will disappear. So this competition does not explain why triangles become smaller in the process of evolution. 

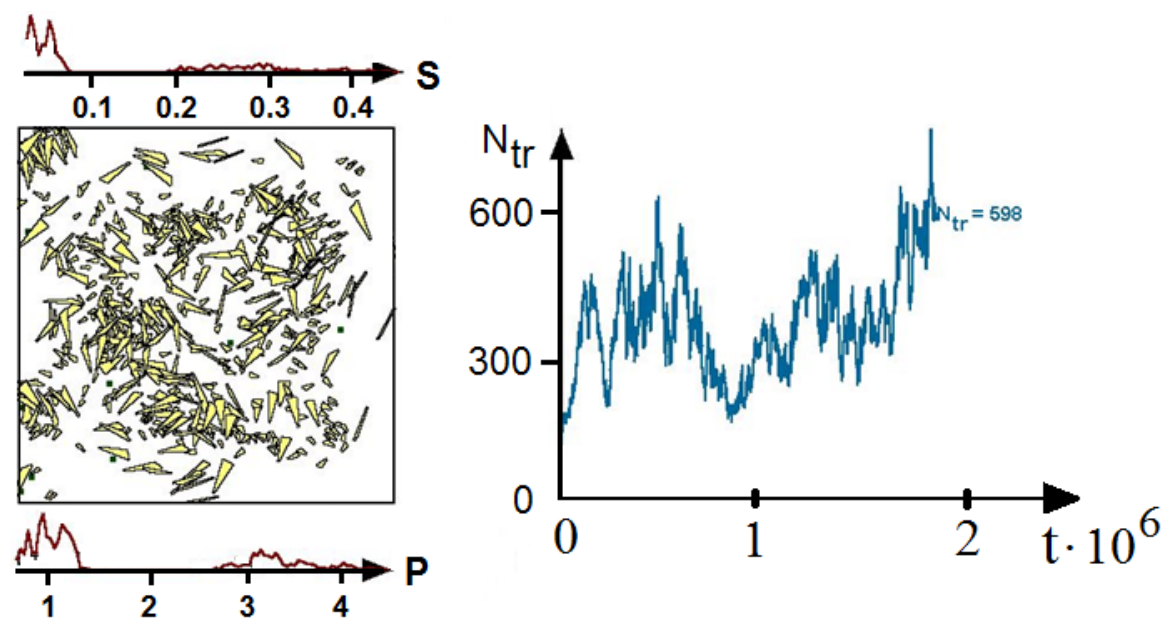

Figure 11: Evolution of triangles $T(1.2,0.3,0.3)$ in the case of food interval adding $\theta=2$. There is a bimodal distribution of triangles with respect to their area $S$ and perimeter $P$. Small triangles continue to decrease and their number increases.

\section{Discussion}

The model developed in this work allows us to study virtual populations of artificial organisms with their genotype, phenotype, mechanism of motion, competition, reproduction. One of the most important feature of these virtual populations is that the fate of their individuals is not prescribed. The artificial organisms recognize food and move to it. Their success is determined by their own properties (morphology) and their environment. The model does not interfere in the process of food search and in the competition with other individuals.

The individuals of the population possess the genotype in the form of three real positive numbers. It determines the phenotype and it is transmitted to offsprings with possible mutations. Moreover, artificial organisms have a mechanism of food recognition, muscles and members that allow them to move towards food, the reproduction system. This seems to be the minimal model of a self-sustained system undergoing Darwinian evolution, which corresponds to the definition of life [16].

We will now compare the behavior of this system with conventional models of population dynamics.

\subsection{Competition of species}

The classical model of competition of species consists of two ordinary differential equations

$$
\begin{aligned}
& \frac{d u}{d t}=k_{1} u(K-a u-b v)-\sigma_{1} u, \\
& \frac{d v}{d t}=k_{2} v(K-c u-d v)-\sigma_{2} v,
\end{aligned}
$$


where $u$ and $v$ are the densities of the two populations which are supposed to be uniformly distributed in space. The first terms in the right-hand sides of these equations describe the reproduction of the populations and the second terms their mortality. The reproduction is proportional to the population density and to available resources, $(K-a u-b v)$ or $(K-$ $c u-d v)$. These terms represent a difference between the rate of appearance of resources $K$ (carrying capacity) and the rate of consumption of resources proportional to the densities of the populations. This model does not take into account the distribution of individuals in the population with respect to phenotype. The model of competition of species with distributed phenotype can be reduced to system (3), (4) under some approximations [25].

The assumption that consumption of resources is proportional to the population density with some given coefficients is very important. It determines the fate of the populations. Depending on these coefficients they can coexist or one of them will disappear losing the competition with the other one. Hence the result of the competition is prescribed by the model. This is the main difference with the approach developed in this work. We introduce individuals with their own properties but we do not know in advance how much resources they will consume. This difference is crucial. The model interferes at the level of initial conditions but not at the level of the results.

On the other hand, observing the populations we can estimate how much resources they consume and use these data as parameters in system (3), (4). Let us try to apply this approach to the results of this work. Equations (3), (4) imply that resources for the first and for the second populations are, generally, different. The coefficients $a, b, c, d$ show how the two types of resources are consumed by the populations. In the case considered in this work, resources for the two populations are the same. They are given as food points presented in the computation domain (Figure 9 ). Hence we set $a=c, b=d$. The two populations have the same resources but possibly different natality and mortality rates.
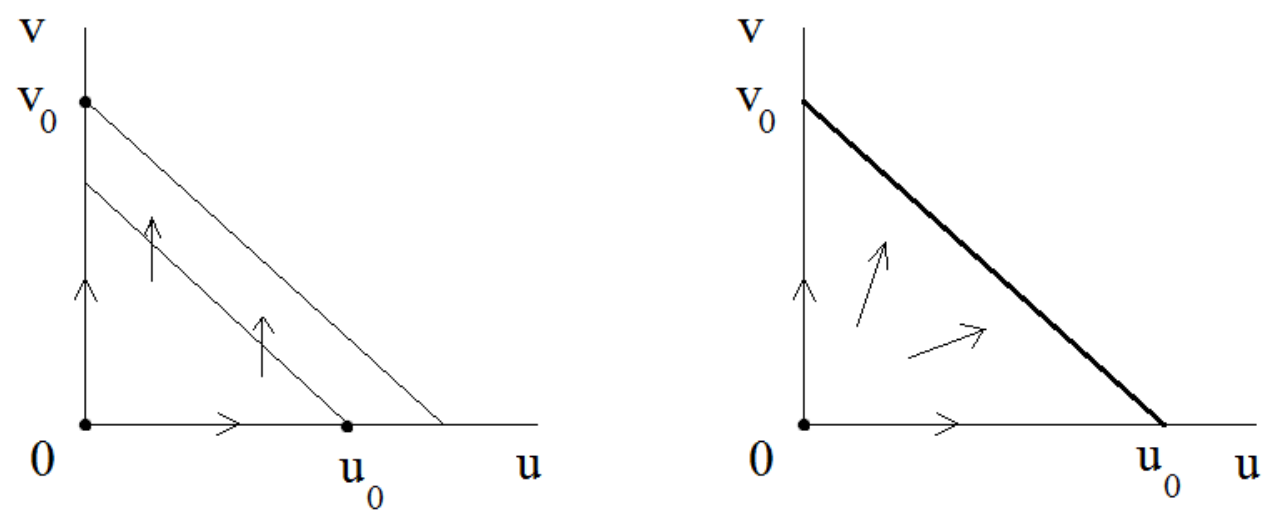

Figure 12: Phase plane of system (3), (4) if $\sigma_{1} / k_{1}>\sigma_{2} / k_{2}$ (left) and $\sigma_{1} / k_{1}=\sigma_{2} / k_{2}$ (right). In the first case there are three isolated stationary points, in the second case the whole interval filled by stationary points (bold line). 
Let us consider the phase plane of system (3), (4). If $\sigma_{1} / k_{1}>\sigma_{2} / k_{2}$, then there are three stationary points: $(0,0),\left(u_{0}, 0\right)$ and $\left(0, v_{0}\right)$, where $u_{0}=K-\sigma_{1} / k_{1}, v_{0}=K-\sigma_{2} / k_{2}$. The first two points are unstable and the last one is stable. If the initial condition $(u(0), v(0))$ is such that $u(0)>0$ and $v(0)$ is positive and sufficiently small, then the trajectory will first approach the saddle point $\left(u_{0}, 0\right)$. It can stay in the vicinity of this point during possibly a long time that depends on the value of $v(0)$. After that it will go away from this point and it will approach the stable point $\left(0, v_{0}\right)$.

This behavior is close to the results observed in Section 3 in the case of unstable coexistence. However, there is an essential difference. Solution of system (3), (4) approaches the unstable point if the component $v(0)$ of the initial condition is sufficiently small. This condition is not necessary for the unstable coexistence in Section 3. Moreover, this coexistence occurs with both species present in some proportion while the second concentration vanish in the the saddle point $\left(u_{0}, 0\right)$.

Unstable coexistence observed in Section 3 became stable when we increased the rate of food supply. In terms of system (3), (4) it corresponds to an increase of $K$. This system possesses stationary points with both positive components only if $\sigma_{1} / k_{1}=\sigma_{2} / k_{2}$ (Figure 12 , right). The stationary points fill the whole interval in this case. They are stable in the sense of Lyapunov though they are not asymptotically stable.

As before, there is certain resemblance with the results of Section 3 where stable coexistence was observed. However, the difference is that the two species are present in some proportion (up to random oscillations) independent of the initial conditions. In the case of system (3), (4) the stationary point to which the trajectory converges is determined by the initial condition.

Thus we can conclude that the system of competition of species captures some properties of the virtual populations but it does not describe their behavior. The reason for this discrepancy is that reproduction of the populations cannot be described in the simple form imposed by this ODE model. The populations are not uniformly distributed in space. They move and compete in a more complex way.

\subsection{Phenotypic evolution}

The results of Section 4 show that populations of artificial organisms can be characterized by their distribution with respect to some morphological parameter. Such distributions can be described by the nonlocal reaction-diffusion equation with global consumption of resources:

$$
\frac{\partial u}{\partial t}=D \frac{\partial^{2} u}{\partial x^{2}}+a u^{k}(K-b I(u))-\sigma u
$$

where

$$
I(u)=\int_{-\infty}^{\infty} u(y, t) d y
$$


These equations are introduced and studied in [5], [25]. They describe evolution of population density distributions in the space of phenotypes. The right-hand side of this equation contains the diffusion term, which describes random mutations, the natality and mortality terms. The integral $I(u)$ replaces $u$ in the logistic equation. It shows that consumption of resources is proportional to the total population taken for all phenotypes. The case $k=1$ corresponds to the asexual reproduction and $k=2$ to sexual reproduction. We consider this equation on the whole axis since the influence of the boundary on the distributions in Figure 8 is negligible.

Solutions of this equation which correspond to the density distribution similar to those shown in Figure 8 are called the pulse solutions. They exist for $k=2$ but not for $k=1$ (if $\sigma$ is constant).
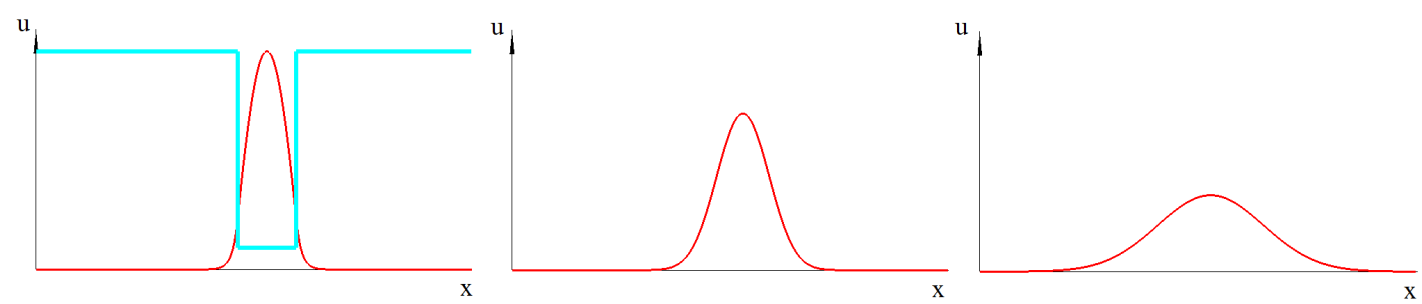

Figure 13: Numerical simulations of equation (5) for $k=1$. In the case of variable $\sigma$ (left image, blue curve), there exists a localized stationary solution. In the case of a constant $\sigma$ there is only trivial stationary solution, $u(x, t)$ converges to zero (middle and right images). The values of parameters: $D=5, a=1, K=2, b=1, \sigma=0.1$ or $\sigma=1$ (right), $\sigma=0.1$ (middle and right).

Let us discuss the case $k=1$ in more detail since it corresponds to the asexual reproduction of artificial organisms considered in this work. It can be proved that in the case of constant $\sigma, I(u) \rightarrow(K-\sigma / a) / b$ and $u(x, t) \rightarrow 0$ uniformly on every bounded interval. This means that that the total population converges to a positive constant but the distribution becomes wider, and for any given phenotype the density distribution function converges to zero.

Suppose now that the mortality coefficient depends on the phenotype. We consider a piece-wise constant function:

$$
\sigma(x)= \begin{cases}\sigma_{0} & , \quad x_{0}<x<x_{1} \\ \sigma_{1} & , \quad x \leq x_{0}, x \geq x_{1}\end{cases}
$$

If $\sigma_{0}$ is sufficiently small and $\sigma_{1}$ is sufficiently large, then there exists a positive stationary solution of equation (5) (Figure 13, left). If we take this solution as the initial condition and set $\sigma \equiv \sigma_{0}$, then the solution converges to zero (Figure 13, middle and right).

Let us return to the evolution of triangles observed in Section 4. Their density distribution with respect to area is localized around some average values (Figure 8). As we discussed above, equation (5) can have localized solutions if the mortality coefficient $\sigma$ is sufficiently 
large outside a finite interval in order to suppress propagation of the population. This is not the case of the artificial organisms considered in this work. The population can exist in a very wide range of phenotypes, much wider than it is really observed. Therefore we can expect that the distribution width increases in time as it is shown in Figures 13 (middle and right). However, triangles with the phenotype strongly different from the principal phenotype are eliminated due to competition. This competition preserves localized distribution of the phenotype of the artificial organisms.

There are two different evolution patterns observed in Section 4. In Section 3 we identified the most efficient triangles. Depending on other parameters, they either win the competition against any other triangles or they coexist with some other triangles. If we take the values of parameters for which the coexistence does not occur, then triangle evolve to the most efficient morphology. If the coexistence is possible in the competition problem without mutations, then in the evolution problem with mutations we also observe two types of triangles (Figure 11). Triangles with the most efficient morphology coexist with another population of triangles whose area decreases and their number increases.

Thus equation (5) captures some features of the observed dynamics (localized solutions) but it does not correctly describe dynamics of these localized solutions. Evolution of the phenotype observed here for the artificial organisms has some specific features determined by the interaction of morphology and evolution.

\subsection{Cope's rule}

Cope's rule suggests that the size of animals increases in the process of the species evolution [20]. Though this assertion does not hold for all species, it is observed for many of them. Its validity and possible explanations are largely debated in literature. Larger body size can increase fitness due to increased capacity to capture prey, to compete with other individuals and for some other reasons. On the other hand, it requires more food and water.

The model developed in this work allows us to make some conclusions about this question. In general we observe that the size of artificial organisms considered here converges to some optimal value. If in the beginning of the evolution it is less than the optimal one, then it will increase. On the other hand, the larger body size will decrease. The evolution of the size of artificial organisms in numerical simulations presented in this work is mainly determined by the competition of two factors. Larger organisms move faster and get more food. Their speed of motion is proportional to their linear size. On the other hand, they need more food to survive and to give offsprings. The required quantity of food is proportional to their mass (area in the 2D case). A complex combination of these and other factors results in the existence of an optimal size.

It appears however that existence of the optimal size depends on parameters of the model. Kleiber's law [15] affirms that animal metabolic rate $\mathrm{q}$ is proportional to a power of mass $M, q \sim M^{n}$, where $n=3 / 4$ (in other data, $n=2 / 3$ ). In our model, we assume that the quantity of required food is proportional to the mass (area) of the organisms. Therefore

$n=1$. In order to verify the influence of this parameter, we modified the model in such a 

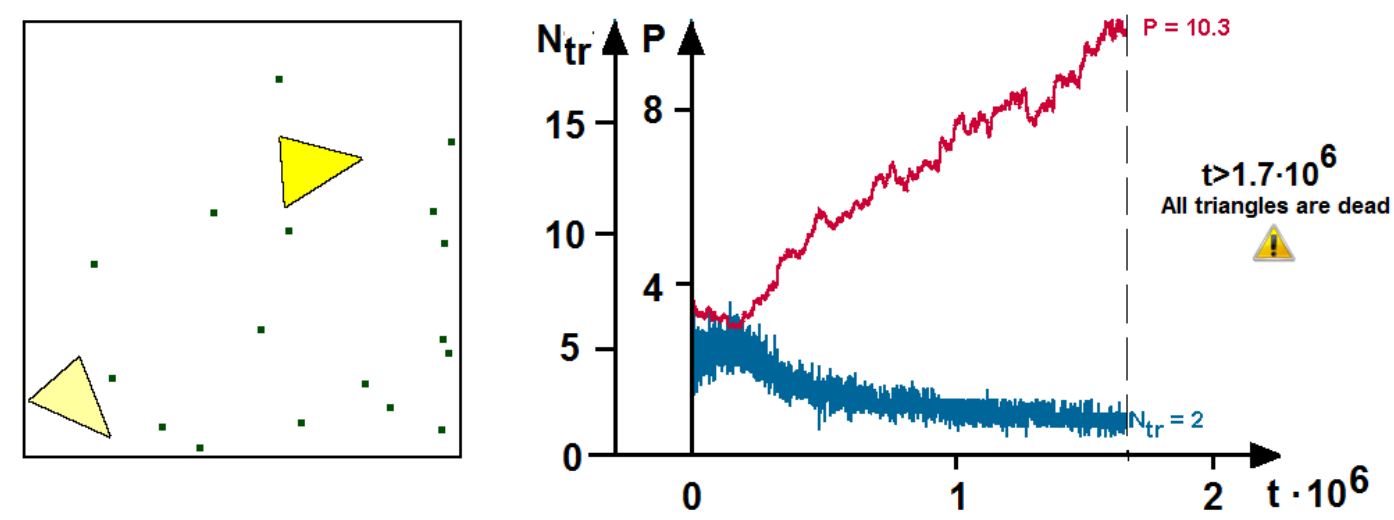

Figure 14: Evolution of triangles in the case where the quantity of required food is proportional to their perimeter. Their size increases, their number decreases and after some time they disappear. Left: a snapshot of computation domain with two triangles and several food points. Right: The evolution of the perimeter of triangles (red curve) and of their number (blue curve) in time.

way that the quantity of required food is proportional to the perimeter of triangles (average linear size) instead of their area. In this case $n=1 / 2$, and the organisms have a tendency to increase (Figure 14). Their perimeter grows and their number decreases. When only few triangles remain, random fluctuations lead to their disappearance. Hence realization of Cope's rule can be related to the value of the exponent in Kleiber's law.

\section{Conclusions}

In this work we study population dynamics of artificial organisms with their genotype, phenotype, mechanism of motion, foraging and reproduction. Their success in the food search and in the reproduction depend on their morphology. More efficient organisms will reproduce more and will transmit their genotype to their offsprings with possible mutations.

We investigated competition of different phenotypes without mutations and their evolution with mutations. When different phenotypes compete with each other, there are more efficient phenotypes that win these competitions. Since direction and the speed of motion of artificial organisms depend on their morphology, then their fitness also depends on morphology. We identified the optimal morphology which allows the artificial organisms to win the competition against the organisms with another morphology.

Mutations during reproduction of artificial organisms allow us to study their evolution. If the initial phenotype is different from the optimal one, the former will rapidly evolve to the latter. Further evolution depends on the values of parameters. In some cases, it is reduced to random oscillations around the optimal phenotype. These oscillations are determined by the competition of different phenotypes. For other values of parameters, another phenotype 
can emerge. The mechanism of this speciation is related to different foraging behaviors of organisms with different morphologies.

Thus, morphology of the artificial organisms can influence their competition and evolution. Dynamics of such populations cannot be described by conventional models of competition and evolution of species because they do not take into account the influence of morphology.

\section{References}

[1] T. Andersen, R. Newman, T. Otter. Shape homeostasis in virtual embryos. Artif. Life, 15 (2009), no. 2, 161-883.

[2] S. Atamas. Self-organization in computer simulated selective systems. Biosystems, 39 (1996), 143-151.

[3] S.A. Baldauf, L. Engqvist, F.J. Weissing. Diversifying evolution of competitiveness. Nature Communications, 2014, 5:5233, DOI: 10.1038/ncomms6233

[4] D. Basanta, M. Miodownik, B. Baum. The evolution of robust development and homeostasis in artificial organisms. PLoS Computational Biology, 4 (2008), no. 3, e1000030.

[5] N. Bessonov, N. Reinberg, V. Volpert. Mathematics of Darwins Diagram. Math. Model. Nat. Phenom., Vol. 9, No. 3, 2014, 5-25.

[6] J. Clune, B.E. Beckmann, C. Ofria, R.T. Pennock. Evolving coordinated quadruped gaits with the HyperNEAT generative encoding. In Proceedings of the 2009 IEEE Congress on Evolutionary Computation (CEC), Trondheim, Norway, 2009.

[7] J.A. Coyne, H.A. Orr. Speciation. Sinauer Associates, Sunderland, 2004.

[8] L. Desvillettes, P.E. Jabin, S. Mischler, G. Raoul. On selection dynamics for continuous structured populations. Commun. Math. Sci., 6 (2008), no. 3, 729-747.

[9] U. Dieckmann, M. Doebeli. On the origin of species by sympatric speciation. Nature 400, 354357 (1999).

[10] S. Gavrilets. Fitness Landscape and the Origin of Species. Princeton University Press, Princeton, 2004.

[11] S. Genieys, V. Volpert, P. Auger. Pattern and waves for a model in population dynamics with nonlocal consumption of resources. Mathem. Modelling of Natural Phenomena, 1, (2006), No. 1, 63-80.

[12] P. Hogeweg. Shapes in the shadow: evolutionary dynamics of morphogenesis. Artif. Life, 6 (2000), 85-101. 
[13] I.A. Johnston, A.F. Bennett. Animals and temperature. Phenotypic and evolutionary adaptation. Cambridge University Press, Cambridge, 1996.

[14] S.A. Kauffman. The origins of order. Self-organization and selection in evolution. Oxford University Press, New York, 1993.

[15] M. Kleiber. Body size and metabolism. Hilgardia 6 (1932), 315-351.

[16] P.L. Luisi. About various definitions of life. Origins of Life and Evolution of the Biosphere, 28 (1998), 613-622.

[17] H. Malchow, S. Petrovskii, E. Venturino. Spatiotemporal patterns in ecology and epidemiology.

[18] A. Matos, R. Suzuki, T. Arita. Heterochrony and artificial embryogeny: a method for analyzing artificial embryogenies based on developmental dynamics. Artif. Life, 15 (2009), no.2, 131-160.

[19] G.F. Oster, N. Shubin, J.D. Murray, P. Alberch. Evolution and morphogeneric rules: the shape of the vertebrate limb in ontogeny and phylogeny. Evolution, 42 (1988), no. $5,862-884$.

[20] B. Rensch. Histological changes correlated with evolutionary changes of body size. Evolution, 2 (1948), no. 3, 218-230.

[21] C.D. Schlihting, M. Pigliucci. Phenotypic evolution: a reaction norm perspective. Sinauer Associates, Sunderland, 1998.

[22] N. Schmidt-Kittler, K. Vogel (Eds.) Constructional morphology and vvolution. SpringerVerlag, Berlin, 1991.

[23] K. Sims. Evolving 3D Morphology and Behavior by Competition. Artificial Life IV Proceedings, ed. by R. Brooks \& P. Maes, MIT Press, 1994, 28-39.

[24] K.O. Stanley, R. Miikkulainen. A taxonomy for artificial embryogeny. Artif. Life, 9 (2003), no. 2, 93-130.

[25] V. Volpert. Elliptic partial differential equations. Volume 2. Reaction-diffusion equations. Birkhäuser, 2014, 784+xviii.

[26] V. Volpert, S. Petrovskii. Reaction-diffusion waves in biology. Physics of Life Reviews, 6 (2009), 267-310. 